\title{
Factors correlated with the improvement of endothelial dysfunction during Abatacept therapy in patients with rheumatoid arthritis
}

This article was published in the following Dove Press journal: Journal of Inflammation Research

\author{
Maurizio Benucci' \\ Francesca Bandinelli' \\ Arianna Damiani' \\ Francesca Li Gobbi' \\ Maria Infantino ${ }^{2}$ \\ Valentina Grossi ${ }^{2}$ \\ Mariangela Manfredi² \\ 'Rheumatology Unit, Hospital S. \\ Giovanni di Dio, Azienda USL-Toscana \\ Centro, Florence, Italy; ${ }^{2}$ Immunology \\ and Allergology Laboratory Unit, \\ Hospital S. Giovanni di Dio, Azienda \\ USL-Toscana Centro, Florence, Italy
}

Correspondence: Maurizio Benucci Rheumatology Unit, Azienda Sanitaria USL-Toscana Centro, Hospital S. Giovanni di Dio, Via Torregalli 3, 50143 Florence, Italy

Tel +390556932636

Fax +390556932099

Email maubenucci@tiscali.it
Background: Rheumatoid arthritis patients are exposed to a high risk of cardiovascular morbidity and mortality even in the early phases of the disease.

Methods: We evaluated carotid common carotid intimal media thickness (ccIMT) intimal thickness and brachial flow-mediated dilation (FMD) of 45 rheumatoid arthritis patients without known cardiovascular risk factors or heart disease on a stable dose of prednisone $5.2 \pm 1.2 \mathrm{mg} /$ day and Methotrexate 11.5 $\pm 2.1 \mathrm{mg}$ at baseline (T0) and after 12 months (T1) of treatment with Abatacept $125 \mathrm{mg} /$ week. The comparison between T0 and T1 ( $t$ - and Mann-Whitney test), correlation (Spearman $r$ ), and predictivity (linear regression) of FMD, ccIMT vs clinical and laboratory parameters (disease activity 28 score, tumor necrosis factor alpha [TNF $\alpha$ ], interleukin-6, erythrocyte sedimentation rate, $\mathrm{C}$-reactive protein (CRP), CD3+, CD3+/CD4+, CD3+/CD8+, CD19+(B), CD20+(B), NK CD3-CD56+CD16+, CD14+HLA DR+, CD4+CD28+, CD4+CD28, rheumatoid factor IgM, IgA, RF IgG, anti-citrullinated peptide antibodies) were also evaluated. Results: During Abatacept treatment, ccIMT and FMD remained stable and disease activity 28 score, CRP, erythrocyte sedimentation rate, and interleukin-6 decreased significantly ( $p=0.0001,0.002,0.0002,0.0001$ respectively). At T0, only ccIMT resulted as correlated with baseline TNF $\alpha$ values $(p=0.0245)$ in an inverse proportion. At T1, ccIMT correlated with CD3/ CD8+ lymphocytes number ( $p=0.0351)$ and FMD with CRP $(p=0.0075)$. In regression analysis, baseline ccIMT and FMD had a low predictivity for TNF $\alpha(p=0.011)$ and CRP $(p=0.049)$ at T1, respectively.

Conclusion: This study shows that the endothelial function remained stable during Abatacept treatment.

Keywords: Abatacept, rheumatoid arthritis, cardiovascular disease

\section{Introduction}

We know from several studies that rheumatoid arthritis (RA) is an important cardiovascular $(\mathrm{CV})$ risk factor in terms of morbidity as well of mortality, and this risk is present even at the beginning of the disease's course. ${ }^{1,2}$ Epidemiological studies have also shown that RA is associated with an increased risk of premature $\mathrm{CV}$ diseases (CVD), including acute myocardial infarction and CV-related mortality. ${ }^{3-7}$ Inflammation plays the main role in this risk assessment, and its effects are involved not only in patients suffering from arthritis but also in the atherogenic process of otherwise healthy people. ${ }^{8-10}$ Endothelial dysfunction (ED) characterizes every step of the atherosclerosis progression, up to the latest stages of vascular disease. Mechanisms leading to ED are pleiotropic, but most of them can be found more expressed in RA patients rather than 
in healthy controls: alterations of nitric oxide's metabolism, increased synthesis of VEGF, chemokines, adhesion cytokines, and ROS production, only to cite the most representative. ${ }^{11}$ Notably, these processes are the same as involved in inflammatory proliferation of synovial vasculature during RA. In order to assess the state of coronary artery endothelium, it is possible to explore its correlation with the brachial artery, which can be investigated in a noninvasive way with the help of vascular ultrasonography. This technique allows to evaluate the endothelium-dependent flow-mediated vasodilatation (FMD). ${ }^{12} \mathrm{ED}$ is a feature shared by patients with longstanding RA treated with Methotrexate, ${ }^{13}$ but an incredibly rapid improvement in FMD can be seen in patients when a treatment with tumor necrosis factor (TNF) blockers is performed. ${ }^{14}$ Conversely, not many data are available regarding RA biological disease modifying drugs (bDMARDs) other than anti-TNF in terms of ED improvement. Two papers (one by Mathieu et al and the other by Sandoo and Kitas) ${ }^{15,16}$ show neither exciting nor comforting results in terms of arterial stiffness in the course of therapy with Abatacept, a soluble cytotoxic T-Lymphocyte Antigen (CTLA)-4Ig fusion protein that blocks CD28-CD80/86 costimulatory T-cell activation, describing a worsening of this parameter.

\section{Materials and methods}

We evaluated in 45 RA (2010 ACR/EULAR classification criteria) patients (mean: 62,75 years old patients [standard error of the mean $\{\mathrm{SEM}\}: 1$ and 7$]$, lower and higher $95 \%$ interval confidence values IC: $61,68-18,32)$. FMD on brachial artery using a probe with $10 \mathrm{MHz}$ transducer (HP Sonos 5500, Hewlett-Packard Development Co., Palo Alto, CA, USA), and common carotid intima media thickness (ccIMT) (10 MHz linear array transducer, HP Sonos 5500) intimal thickness at baseline and after 12 months of treatment with Abatacept $125 \mathrm{mg} /$ week. Patients were on a stable dose of prednisone $5.2 \pm 1.2 \mathrm{mg} /$ day and Methotrexate $11.5 \pm 2.1 \mathrm{mg} /$ week, did not have significant changes in CV factors (no cerebral and CV events), and had stable arterial pressure during the follow-up, and did not have diabetes, smoking habit, and obesity at baseline. Furthermore, demographics (gender and illness duration) were considered in the statistical analysis.

We did not analyze patients switched to other treatments (DMARDs and bDMARDs) because the time of washout of Abatacept because in the short term it could influence the parameters evaluated. Because of the retrospective nature of the study and possible other $\mathrm{CV}$ confounding factors in the control population, we could not compare this data with another cohort.
Patients have also been evaluated for disease activity 28 score (DAS28) and for the following laboratory parameters: TNF $\alpha$ (Human TNF-alpha Quantikine Immunoassay; R\&D Systems Inc, Minneapolis, MN, USA); interleukin (IL)-6 (Human IL-6 Instant Enzyme-linked Immunosorbent assay; eBioscience, Bender MedSystem GmbH, Vienna, Austria); the erythrocyte sedimentation rate (ESR) and/or C-reactive protein (CRP) levels (Unicel Coulter DxC 800 Synchron Central System; Beckman Coulter Inc, Brea, CA, USA); $\mathrm{CD} 3+, \mathrm{CD} 3+/ \mathrm{CD} 4+, \mathrm{CD} 3+/ \mathrm{CD} 8+, \mathrm{CD} 19+(\mathrm{B}), \mathrm{CD} 20+(\mathrm{B})$, NK CD3-CD56+CD16+, CD14+ HLA DR+, CD4+CD28+, CD4+CD28 null peripheral mononuclear cells (BD FACSCanto II flow cytometer; Biosciences, San Jose, CA, USA); rheumatoid factor (RF) IgM (N Latex RF, Siemens AG, Munich, Germany); RF IgA and RF IgG (Enzyme Immuno Assay Orgentec Diagnostika GmbH, Mainz, Germany); anti-citrullinated peptide antibodies (ACPA; Anti-CCP AxisShield Diagnostics Ltd, Luna Place, The Technology Park, Dundee, DD2 1XA, UK). All patients gave their written informed consent according to the retrospective nature of the study according to the Declaration of Helsinki and Italian legislation (Authorization of the Privacy Guarantor No. 9, December 12, 2013). The Institutional Review Board, the Health Director of San Giovanni di Dio Hospital in Florence, reviewed and approved this research and the use of clinical and laboratory data of common clinical practice, in the respect of the Privacy Law, for clinical and scientific studies and publications.

\section{Assessment of brachial artery FMD and ccIMT}

A single trained sonographer estimated, with a $10-\mathrm{MHz}$ linear ultrasound probe (HP Sonos 5500; Hewlett-Packard Development Co.), common carotid artery for ccIMT and brachial artery for FMD, at baseline (before the first infusion) and after 12 months. ccIMT measurements were obtained with patients lying supine, the neck extended, and the chin directed to the side opposite to the examined one. Considering that we indicate as IMT the distance between the leading edges of the lumen interfaces and the media-adventitia interface of the far wall and knowing that in ultrasonography this distance can be assessed measuring the space between the first and the second echogenic lines from the lumen, both carotid arteries were delaminated three times, $1 \mathrm{~cm}$ distal to the carotid bifurcation, at the level of the posterior wall, expressed in millimeters. The average of these measures was taken into account as an indicator of carotid vascular stiffness. Although 
any normal range has already been established, an increase of $>1 \mathrm{~mm}$ was considered abnormal. ${ }^{17}$

For FMD, patients had rested previously for 30 minutes in a temperature-controlled room. Then, a B-mode longitudinal section of the right arm brachial artery was evaluated above the antecubital fossa. A pneumatic cuff was positioned on the forearm, inflated to an over-systolic pressure for 4.5 minutes and then released with the aim of inducing reactive hyperemia. After deflation, the maximal flow velocity and arterial diameter were recorded for 90 minutes, ECG-gated, and detected offline. The FMD measurement was performed at baseline (before the first infusion) and repeated after 12 months.

\section{Statistical analysis}

Descriptive statistics were expressed as mean \pm SEM, 95\% lower and upper confidence intervals of mean, and as number and percentage for categorical variables. A $p$-value $<0.05$ was considered statistically significant. Normal distribution of parameters was verified by "KS normality test" and "Wilcoxon Signed Rank Test".

T0 and T1 parameters were compared with parametric $t$-test or nonparametric Mann-Whitney, when indicated. The correlation of FMD and ccIMT with other parameters at T0 and T1 was evaluated with nonparametric Pearson and parametric Spearman $r$ test, respectively, when indicated. Finally, predictive values of T0 FMD and ccIMT for other parameters at $\mathrm{T} 1$ were analyzed with linear regression, for the absence of other CV bias factors as illustrated in methods.

\section{Results}

In the 45 RA patients examined, ccIMT and FMD T0 and T1 were independent by gender ( 32 female vs 13 male) and
RA disease duration (5.7 years [0.53 SEM], 4.6-6.8 95\% CI) at statistical analysis (Mann-Whitney and Spearman $r$ test, respectively). During treatment, ccIMT and FMD remained stable, with a low increase in FMD from T0 to T1 (+1.35\%). Following Abatacept treatment, DAS28, CRP, ESR, and IL-6 significantly decreased by $23.55 \%, 38.90 \%, 29.4 \%$, and $60.66 \%$, respectively (percentage expressed over T0 values) with significant values shown in Table 1 . At T0, only ccIMT resulted as correlated with baseline TNF $\alpha$ values $(p=0.0245)$ in an inverse proportion (data not shown) and not with other evaluated parameters. Baseline FMD does not correlate with any of the parameters considered. At T1, after 12 months of Abatacept $125 \mathrm{mg} /$ week treatment, FMD correlated with CRP ( $p=0.0075)$, while ccIMT shows correlation with the number of CD3+/CD8+lymphocytes $(p=0.0351)$ and TNF $\alpha$ $(p=0.0299)$, both evaluated after treatment (Table 2$)$. In regression analysis the baseline levels of ccIMT and FMD correlated with TNF $\alpha$ levels $(p=0.011)$, while at the time T1 with CRP levels $(p=0.049)$.

Table I Significant comparison between baseline and follow-up parameters analyzed before and after treatment with Abatacept in RA patients

\begin{tabular}{llll}
\hline & TO & T I & p-statistics \\
\hline ESR & $29.82(1.9)$, & $21.07(2.2), 16.5 I-25.63$ & $0.0002(\mathrm{MW})$ \\
& $25.99-33.65$ & & \\
CRP & $1.37(0.1), 1.04-1.70$ & $0.83(0.12), 0.57-1.1$ & $0.0023(\mathrm{MW})$ \\
DAS28 & $3.79(0.10), 3.57-4$ & $2.89(0.15), 2.59-3.20$ & $<0.000$ I (TT) \\
IL-6 & I0.22(2.08), & $4.02(0.37), 3.25-4.78$ & $<0.000$ I (TT) \\
& $6.01-14.43$ & & \\
\hline
\end{tabular}

Note: Values are expressed in mean, SEM, and $95 \% \mathrm{Cl}$.

Abbreviations: CRP $(\mathrm{mg} / \mathrm{dL})$, C-reactive protein; DAS28, disease activity 28 score; ESR $(\mathrm{mm} / \mathrm{h})$, erythrocyte sedimentation rate; IL-6 $(\mathrm{pg} / \mathrm{mL})$, interleukin-6; MW, Mann-Whitney test; RA, rheumatoid arthritis; SEM, standard error mean; TT, Student's t-test.

Table 2 Significant correlation of FMD and ccIMT at baseline (T0) and at I year follow-up (TI) with other parameters at T0 and TI, respectively

\begin{tabular}{|c|c|c|c|c|c|}
\hline & & FMD TO & ccIMT TO & FMD TI & ccIMT TI \\
\hline & & $\begin{array}{l}5.52(0.04) \\
5.42-5.61\end{array}$ & $\begin{array}{l}0.77(0.01) \\
0.74-0.80\end{array}$ & $\begin{array}{l}5.604(0.04) \\
5.50-5.70\end{array}$ & $\begin{array}{l}0.74(0.00 I), \\
0.7 I-0.77\end{array}$ \\
\hline CRP $(\mathrm{mg} / \mathrm{dL}) \mathrm{T0}$ & $1.37(0.1), 1.04-1.70$ & Not significant & Not significant & - & - \\
\hline CRP TI & $0.83(0.12), 0.57-1.1$ & - & - & $p=0.0075$ & Not significant \\
\hline TNF $\alpha$ T0 & 21.49 (3.6), $14.14-28.84$ & Not significant & $p=0.02$ & - & - \\
\hline TNF $\alpha$ TI & $25.80(6.09), 6.01-14.43$ & - & - & Not significant & $p=0.02$ \\
\hline CD3/CD8+T0 & $514.3(42.6), 428.5-600.2$ & Not significant & Not significant & - & - \\
\hline CD3/CD8+TI & 500.2 (35.53), 428.6-571.9 & - & - & Not significant & $p=0.03$ \\
\hline
\end{tabular}

Note: Values are expressed in mean, SEM, and $95 \% \mathrm{Cl}$.

Abbreviations: CRP (mg/dL), C-reactive protein; FMD, flow-mediated dilation; SEM, standard error mean; TNF $\alpha$ (pg/mL), tumor necrosis factor alpha; cclMT, common carotid intima media thickness. 


\section{Discussion}

Although our study has important limitations, such as its retrospective nature, that do not allow the availability of a control group and the possibility of a bias in assessing $\mathrm{CV}$ risk driven by the elevated age of patients examined, data in our possession represent, in the light of our knowledge, the first evidence of Abatacept's safety in terms of ED. In fact, a previous study by Mathieu et al ${ }^{15}$ found a worsening in arterial stiffness evaluated by pulse wave velocity (PWV) during the treatment with this drug, rising concerns about its role in the fragile equilibrium of RA patients' vasculature. Also Sandoo et al, ${ }^{16}$ in a letter to the editor, described a negative effect on both micro and large vessels' endothelial function in a 32-year-old woman affected by rheumatoid arthritis and treated with Abatacept. By contrast, Abatacept's pharmacodynamical activity suggests stimulating speculations regarding its possible incisive role in controlling both the rheumatic disease and the $\mathrm{CV}$-associated comorbidity. In fact, it is known that rheumatoid arthritis can lead to accelerated atherogenesis by promoting endothelial dysfunction, following the activation of the cytokine system. A healthy endothelium lies in a state that can be defined as antiinflammatory, antithrombotic, anticoagulant, profibrinolytic, and anti-proliferative. ${ }^{18}$ Also, this homeostatic condition depends on refined and sensitive regulatory pathways, and it can easily be perturbed and transformed into the opposite-featured condition identified as "endothelial dysfunction". Atherosclerosis is actually a chronic inflammatory disease in which endothelial alteration leads to retention of oxidized low-density lipoprotein cholesterol particles into the intimal artery layer, attracting leukocytes and causing a localized inflammatory reaction. ${ }^{19}$ Once oxidized, LDL cholesterol particles become neoantigens whose T-cells are able to respond thanks to the signals provided by peptide-major histocompatibility complex (MHC) antigen complexes on antigen-presenting cells. MHC complexes bind the T-cell antigen receptor, and, along with costimulatory signals, this leads to an inflammatory activation. Resting T-cells constitutively express the dominant costimulatory receptor $\mathrm{CD} 28$, whereas activated T-cells are characterized by the expression of the coinhibitory CTLA-4 receptor. ${ }^{20}$ In light of the substantial intravascular CTLA-4+ T-cell infiltration found in samples while investigating the course of the postinterventional remodeling process, Ewing et al used a femoral artery cuff mouse model to elucidate the role of T-cells and the CD28-CD80/86 costimulatory and CTLA-4 coinhibitory pathways in this context. CD4-/- and CD80-/-CD86-/- development of mice intimal lesions was reduced in comparison to normal C57B1/6J controls. Data are available showing that systemic Abatacept treatment, a soluble CTLA-4Ig fusion protein that blocks CD28-CD80/86 costimulatory T-cell activation, can prevent intimal thickening by $58.5 \% .{ }^{21}$ Being an inflammatory disease, atherosclerosis accounts for several cytokines involved in its pathogenesis. The role of IL-6 in the development of CV disease has been deeply investigated. There is evidence that genetic variations in the $I L-6$ gene and its receptor gene (IL-6R) lead to different immune responses, and they are associated with predisposition to CVD. In brief, the IL-6 polymorphism genotypes are mainly associated with proinflammatory cytokines, whereas the IL-6R polymorphism genotypes are associated with antiinflammatory cytokines. ${ }^{22,23}$ Moreover, people with established CAD can host different cytokines subsets, confirming the importance of the genetic contribution in CAD. ${ }^{24}$ CTLA4-Ig is able to modulate macrophagic cytokine expression (TNF $\alpha$ and IL-6) probably acting on NF-kB pathway. This effect, which can also be encountered in vitro on activated human macrophages, is stronger using CTLA4-Ig-DEX and CTLA4-Ig-DEX-MTX combined treatments rather than monotreatment. In particular, a decrease of cytoplasmic NF-kB expression can be observed along with an increase of NF-kB inhibitor, IKB $\alpha .{ }^{25,26}$ In our attempt to discover correlation between markers of inflammation (CRP, ESR, IL-6, and TNF $\alpha$ ) and measures of arterial stiffness (FMD and ccIMT), we did not have statistically significative data, except for the unexpected negative correlation between TNF $\alpha$ levels and ccIMT. When we reassessed these parameters after 12 months of treatment with Abatacept, we detected a significant reduction in both laboratory (CRP, ESR, IL-6, and TNF $\alpha$ ) and clinimetric indexes (DAS28) of inflammation. A nonsignificant reduction was found in ccIMT, along with a slightly increased FMD. Surprisingly, the improvement of FMD positively correlated with followup levels of CRP while ccIMT showed correlation with the number of CD3+/CD8+ lymphocytes and TNF $\alpha$, both evaluated after treatment. Moreover, in regression analysis, we detected that $\mathrm{T} 0$ value of ccIMT can be predictive of TNF $\alpha$ at follow-up $(p=0.011)$, maybe indicating a higher resistance against therapy in patients with more severe arterial stiffness at baseline.

Also, the high average age of our population should be taken into account in the evaluation of TNF $\alpha$ levels both at baseline and at follow-up. In fact, this parameter tends to increase with aging, independently from any other morbidity, and its amount can fluctuate without true clinical significance. On the other hand, FMD value at $\mathrm{T} 0$ was predictive of CRP levels at T1 $(p=0.049)$, confirming the controversial 
relationship between these two parameters, emerging at follow-up. The lack of a clear relationship between routine laboratory parameters of inflammation and arterial stiffness, in the face of their parallel comprehensive improvement at $\mathrm{T} 1$, may suggest the role of other unexplored inflammation mediators in this complex dialogue.

Our findings are in contrast with the ones noted by Mathieu et al, ${ }^{15}$ although in their case, analysis of arterial stiffness was made through PWV and not with FMD; neither measurement of ccIMT was performed; authors of that study assume the lack of total control on inflammation as a possible reason for their results, without excluding the role of an increase of total cholesterol levels. Indeed, PWV got worse only in patients who did not reach disease remission, while it remained stable in the group in which the treatment achieved success. Elevation of levels of lipids is largely described in the course of bDMARDs therapies, although their effect on the progression of atherogenesis remains controversial. Tocilizumab, a monoclonal antibody against IL-6 receptor available in RA treatment, accounts for the most debated concerns in this field, but latest studies seemed to be reassuring about CV safety of this drug. ${ }^{27-29}$ Data are also available about anti-TNF $\alpha$ being discordant as to its effect on lipidic profile, whereas the improvement of CV risk with this class of drug is well known. ${ }^{30}$ We did not assess lipidic profile in our study, whereas Sidiropoulos et $\mathrm{al}^{31}$ analyzing the variation of FMD and ccIMT of 21 RA patients undergoing 18 months of anti-TNF $\alpha$ treatment described a significant improvement of FMD without changes to the ccIMT - agreeing with our findings - with no correlation with lipidic levels. From what we observed, FMD seems to be the most variable and modifiable parameter regarding vascular stiffness, while ccIMT appears to be less plastic. This could reflect a more chronic and irreversible process on the basis of increasing intimal thickness. Furthermore, Spinelli et $\mathrm{al}^{32}$, in a study conducted to evaluate the role of classic and novel RA autoantibodies in determining $\mathrm{CV}$ risk, noted that RA patients have an FMD lower than healthy controls but they lack divergence in terms of ccIMT. So FMD could be burdened by higher pathogenic power.

In conclusion, this study confirms that T-cell costimulation through the CD28-CD80/86 pathway may play a role in accelerated atherosclerosis development observed in patients with chronic inflammatory diseases, and it could be negatively regulated by CTLA-4 coinhibition. Data on CD3+/CD8+ lymphocytes' correlation with ccIMT at T1 could also deserve investigation to assess the role of this cellular population in vascular damage. Overall, our results may also dispel concerns about using Abatacept in RA patients suffering from $\mathrm{CV}$ disease: endothelial parameters do not worsen in the course of treatment, while the improvement in joint and systemic inflammation could help in reducing atherogenesis and CV risk. Further studies could be helpful in better understanding which mediators have the deepest involvement in being a molecular bridge between RA and atherosclerosis.

\section{Disclosure}

The authors report no conflicts of interest in this work.

\section{References}

1. Kremers HM, Crowson CS, Therneau TM, Roger VL, Gabriel SE. High ten-year risk of cardiovascular disease in newly diagnosed rheumatoid arthritis patients: a population-based cohort study. Arthritis Rheum. 2008;58(8):2268-2274.

2. Aviña-Zubieta JA, Choi HK, Sadatsafavi M, Elminan M, Esdaile JM, Lacaille D. Risk of cardiovascular mortality in patients with rheumatoid arthritis: a meta-analysis of observational studies. Arthritis Rheum. 2008;59(12):1690-1697.

3. Solomon DH, Goodson NJ, Katz JN, et al. Patterns of cardiovascular risk in rheumatoid arthritis. Ann Rheum Dis. 2006;65(12):1608-1612.

4. Maradit-Kremers H, Nicola PJ, Crowson CS, Ballman KV, Gabriel SE. Cardiovascular death in rheumatoid arthritis: a population-based study. Arthritis Rheum. 2005;52(3):722-732.

5. Solomon DH, Karlson EW, Rimm EB, et al. Cardiovascular morbidity and mortality in women diagnosed with rheumatoid arthritis. Circulation. 2003;107(9):1303-1307.

6. del Rincón ID, Williams K, Stern MP, Freeman GL, Escalante A. High incidence of cardiovascular events in a rheumatoid arthritis cohort not explained by traditional cardiac risk factors. Arthritis Rheum. 2001;44(12):2737-2745.

7. Wolfe F, Michaud K. The risk of myocardial infarction and pharmacologic and nonpharmacologic myocardial infarction predictors in rheumatoid arthritis: a cohort and nested case-control analysis. Arthritis Rheum. 2008;58(9):2612-2621.

8. Del Rincón I, Williams K, Stern MP, Freeman GL, O’Leary DH, Escalante A. Association between carotid atherosclerosis and markers of inflammation in rheumatoid arthritis patients and healthy subjects. Arthritis Rheum. 2003;48(7):1833-1840.

9. Goodson NJ, Symmons DP, Scott DG, Bunn D, Lunt M, Silman AJ. Baseline levels of $\mathrm{C}$-reactive protein and prediction of death from cardiovascular disease in patients with inflammatory polyarthritis: a ten-year followup study of a primary care-based inception cohort. Arthritis Rheum. 2005;52(8):2293-2299.

10. Van Doornum S, McColl G, Wicks IP. Accelerated atherosclerosis: an extraarticular feature of rheumatoid arthritis? Arthritis Rheum. 2002;46(4):862-873.

11.Yang X, Chang Y, Wei W. Endothelial dysfunction and inflammation: immunity in rheumatoid arthritis. Mediators Inflamm. 2016;2016:6813016.

12. Vita JA, Keaney JF Jr. Endothelial function: a barometer for cardiovascular risk? Circulation. 2002;106(6):640-642.

13. Gonzalez-Juanatey C, Testa A, Garcia-Castelo A, et al. HLA-DRB1 status affects endothelial function in treated patients with rheumatoid arthritis. Am J Med. 2003;114(8):647-652.

14. Gonzalez-Juanatey C, Testa A, Garcia-Castelo A, Garcia-Porrua C, Llorca J, Gonzalez-Gay MA. Active but transient improvement of endothelial function in rheumatoid arthritis patients undergoing longterm treatment with anti-tumor necrosis factor alpha antibody. Arthritis Rheum. 2004;51(3):447-450. 
15. Mathieu S, Couderc M, Glace B, et al. Effects of 6 months of abatacept treatment on aortic stiffness in patients with rheumatoid arthritis. Biologics.2013;7:259-264.

16. Sandoo A, Kitas GD. The impact of abatacept treatment on the vasculature in patients with rheumatoid arthritis. Clin Exp Rheumatol. 2015;33(4):589

17. Kerekes G, Szekanecz Z, Dér H, et al. Endothelial dysfunction and atherosclerosis in rheumatoid arthritis: a multiparametric analysis using imaging techniques and laboratory markers of inflammation and autoimmunity. J Rheumatol. 2008;35(3):398-406.

18. Totoson P, Maguin-Gaté K, Prati C, Wendling D, Demougeot C. Mechanisms of endothelial dysfunction in rheumatoid arthritis: lessons from animal studies. Arthritis Res Ther. 2014;16(1):202.

19. Hansson GK, Hermansson A. The immune system in atherosclerosis. Nat Immunol. 2011;12(3):204-212.

20. Gotsman I, Sharpe AH, Lichtman AH. T-cell costimulation and coinhibition in atherosclerosis. Circ Res. 2008;103(11):1220-1231.

21. Ewing MM, Karper JC, Abdul S, et al. T-cell co-stimulation by CD28CD80/86 and its negative regulator CTLA-4 strongly influence accelerated atherosclerosis development. Int J Cardiol. 2013;168(3):1965-1974.

22. Mitrokhin V, Nikitin A, Brovkina O, et al. Association between interleukin-6/6R gene polymorphisms and coronary artery disease in Russian population: influence of interleukin-6/6R gene polymorphisms on inflammatory markers. J Inflamm Res. 2017;10:151-160.

23. Ma H, Sun $\mathrm{G}$, Wang $\mathrm{W}$, et al. Association between Interleukin-6-572 $\mathrm{C}>\mathrm{G}$ and $-174 \mathrm{G}>\mathrm{C}$ polymorphisms and hypertension: a meta-analysis of case control studies. Medicine (Baltimore). 2016;95(2):e2416.

24. Zhang LL, Wang YQ, Fu B, Zhao SL, Kui Y. Aldehyde dehydrogenase 2 (ALDH2) polymorphism gene and coronary artery disease risk: a meta-analysis. Genet Mol Res. 2015;14(4):18503-18514.
25. Cutolo M, Paolino S, Pizzorni C, et al. Effects of combined treatments with CTLA4-IG (abatacept), dexamethasone and methotrexate on cultured human macrophages. Clin Exp Rheumatol. 2016;34(3): 500-506.

26. Cutolo M, Soldano S, Contini P, et al. Intracellular NF-kB-decrease and IKB $\alpha$ increase in human macrophages following CTLA4-Ig treatment. Clin Exp Rheumatol. 2013;31(6):943-946.

27. Kume K, Amano K, Yamada S, Hatta K, Ohta H, Kuwaba N. Tocilizumab monotherapy reduces arterial stiffness as effectively as etanercept or adalimumab monotherapy in rheumatoid arthritis: an open-label randomized controlled trial. J Rheumatol. 2011;38(10): 2169-2171.

28. Protogerou AD, Zampeli E, Fragiadaki K, Stamatelopoulos K, Papamichael C, Sfikakis PP. A pilot study of endothelial dysfunction and aortic stiffness after interleukin-6 receptor inhibition in rheumatoid arthritis. Atherosclerosis. 2011;219(2):734-736.

29. Kim SC, Solomon DH, Rogers JR, et al. Cardiovascular safety of Tocilizumab versus tumor necrosis factor inhibitors in patients with rheumatoid arthritis: a multi-database cohort study, Arthritis Rheumatol. 2017;69(6):1154-1164.

30. Popa C, Netea MG, Radstake T, et al. Influence of anti-tumour necrosis factor therapy on cardiovascular risk factors in patients with active rheumatoid arthritis. Ann Rheum Dis. 2005;64(2):303-305.

31. Sidiropoulos PI, Siakka P, Pagonidis K, et al. Sustained improvement of vascular endothelial function during anti-TNFa treatment in rheumatoid arthritis patients. Scand J Rheumatol. 2009;38(1):6-10

32. Spinelli FR, Pecani A, Ciciarello F et al. Association between antibodies to carbamylated proteins and subclinical atherosclerosis in rheumatoid arthritis patients. BMC Musculoskelet Disord.2017;18:214.
Journal of Inflammation Research

\section{Publish your work in this journal}

The Journal of Inflammation Research is an international, peer-reviewed open access journal that welcomes laboratory and clinical findings on the molecular basis, cell biology and pharmacology of inflammation including original research, reviews, symposium reports, hypothesis formation and commentaries on: acute/chronic inflammation; mediators of

\section{Dovepress}

inflammation; cellular processes; molecular mechanisms; pharmacology and novel anti-inflammatory drugs; clinical conditions involving inflammation. The manuscript management system is completely online and includes a very quick and fair peer-review system. Visit http://www.dove press.com/testimonials.php to read real quotes from published authors. 